\title{
Associação entre nível de atividade física e síndrome metabólica em pacientes vivendo com HIV e em tratamento antirretroviral no Município de Ponta Grossa
}

\author{
Association between physical activity level and metabolic syndrome in patients living with HIV and \\ in antiretroviral treatment in the City of Ponta Grossa \\ Asociación entre nivel de actividad física y síndrome metabólico en pacientes viviendo con YIH y en \\ tratamiento antirretroviral en la Ciudad de Ponta Grossa
}

Recebido: 04/03/2021 | Revisado: 10/03/2021 | Aceito: 13/03/2021 | Publicado: 20/03/2021

\author{
Lucas Fernando Fonseca \\ ORCID: https://orcid.org/0000-0002-1774-6576 \\ Universidade Estadual de Ponta Grossa, Brasil \\ E-mail: lffonsecac31@gmail.com \\ Camila Marinelli Martins \\ ORCID: https://orcid.org/0000-0002-6430-2687 \\ Universidade Estadual de Ponta Grossa, Brasil \\ AAC\&T Consultoria em Pesquisa LTDA, Brasil \\ E-mail: cami.marinelli@gmail.com \\ Diego Osmar Rodrigues \\ ORCID: https://orcid.org/0000-0001-8451-5439 \\ Universidade Estadual de Ponta Grossa, Brasil \\ E-mail: diegoosmar.rodrigues@hotmail.com \\ Ricardo Zanetti Gomes \\ ORCID: https://orcid.org/0000-0002-9651-8298 \\ Universidade Estadual de Ponta Grossa, Brasil \\ E-mail: zanetticons@uol.com.br \\ Erildo Vicente Muller \\ ORCID: https://orcid.org/0000-0003-4643-056X \\ Universidade Estadual de Ponta Grossa, Brasil \\ E-mail: zanetticons@uol.com.br
}

\begin{abstract}
Resumo
No Brasil, até 2018, existiam, num acumulado, 926.742 pessoas vivendo com HIV/AIDS (PVHIV). Os antirretrovirais (ARV's) são capazes de controlar o vírus e melhorar a qualidade de vida destes indivíduos, porém, o uso destas drogas pode levar ao desenvolvimento da síndrome metabólica (SM). Sabe-se que as atividades físicas são fatores protetores para SM em pessoas saudáveis, porém, em pacientes em tratamento por ARV's, esta associação ainda não é clara. Objetivo: identificar se a atividade física é fator protetor para SM em (PVHIV) em uso de ARVs. Método: estudo transversal, quantitativo com 265 (PVHIV) e em tratamento com ARV's no serviço de assistência especializada (SAE) de Ponta Grossa-PR entre março/2018 e maio/2019. O nível de atividade física foi medido com o questionário IPAQ versão curta. Os dados foram analisados de maneira descritiva e a associação entre SM e atividade física foi medida com OR (odds ratio) e qui-quadrado. Resultados: a média de idade foi de 43 anos, 56,2\% eram do sexo biológico masculino, $61,9 \%$ de etnia branca, $71,3 \%$ heterossexuais, $44,5 \%$ com ensino fundamental, $41,1 \%$ com renda de 1-2 salários mínimos, 40,4\% casados e em 74,7\% o modo de transmissão foi sexual. Quanto a atividade física, 57,0\% eram ativos ou irregularmente ativos e 67,5\% não apresentavam SM. Entre os ativos, 31,1\% tinham SM e entre os inativos $34,2 \%$, o que não configurou associação estatisticamente significativa (OR 0,87; IC 95\% 0,52 1,46; $\mathrm{p}=0,595$ ). Conclusão: Sob essa perspectiva, a atividade física não foi fator protetor para SM em (PVHIV) em uso terapias antirretrovirais (TARVs).
\end{abstract}

Palavras-chave: AIDS; Dislipidemias; Terapia antirretroviral; Exercício físico.

\begin{abstract}
In Brazil, until 2018, there were, in an accumulated number, 926,742 people living with HIV / AIDS (PLHIV). Antiretrovirals (ARV's) are able to control the virus and improve the quality of life of these individuals, however, the use of these drugs can lead to the development of metabolic syndrome (MS). It is known that physical activities are protective factors for MS in healthy people, however, in patients undergoing treatment for ARVs, this association is still unclear. Objective: to identify whether physical activity is a protective factor for MS in (PLHIV) using ARVs. Method: cross-sectional, quantitative study with 265 (PLHIV) and undergoing treatment with ARVs in the specialized assistance service (SAE) of Ponta Grossa-PR between March / 2018 and May / 2019. The level of physical activity
\end{abstract}


was measured using the IPAQ short version questionnaire. The data were analyzed descriptively and the association between MS and physical activity was measured with OR (odds ratio) and chi-square. Results: the average age was 43 years, $56.2 \%$ were male, $61.9 \%$ white, $71.3 \%$ heterosexual, $44.5 \%$ with elementary education, $41.1 \%$ with income of 1-2 minimum wages, $40.4 \%$ married and in $74.7 \%$ the mode of transmission was sexual. As for physical activity, $57.0 \%$ were active or irregularly active and $67.5 \%$ did not have MS. Among the active, $31.1 \%$ had MS and among the inactive $34.2 \%$, which did not represent a statistically significant association (OR 0.87; 95\% CI $0.52-1.46$; p = 0.595). Conclusion: From this perspective, physical activity was not a protective factor for MS in (PLHIV) using antiretroviral therapies (ARV).

Keywords: AIDS; Dyslipidemias; Antiretroviral therapy; Physical exercise.

\section{Resumen}

En Brasil, hasta 2018, había, en número acumulado, 926,742 personas viviendo con VIH / SIDA (PVVIH). Los antirretrovirales (ARV) son capaces de controlar el virus y mejorar la calidad de vida de estos individuos, sin embargo, el uso de estos medicamentos puede conducir al desarrollo del síndrome metabólico (EM). Se sabe que la actividad física es un factor protector para la EM en personas sanas, sin embargo, en pacientes sometidos a tratamiento con ARV, esta asociación aún no está clara. Objetivo: identificar si la actividad física es un factor protector para la EM en (PVVIH) que usan ARV. Método: estudio transversal, cuantitativo con 265 (PVVIH) y en tratamiento con ARV en el Servicio de Atención Especializada (SAE) de Ponta Grossa-PR entre marzo / 2018 y mayo / 2019. El nivel de actividad física se midió mediante el cuestionario IPAQ versión corta. Los datos se analizaron de forma descriptiva y la asociación entre SM y actividad física se midió con OR (odds ratio) y chi-cuadrado. Resultados: la edad promedio fue de 43 años, $56.2 \%$ eran hombres, $61.9 \%$ blancos, $71.3 \%$ heterosexuales, $44.5 \%$ con educación básica, $41.1 \%$ con ingresos de 1-2 salarios mínimos, $40.4 \%$ casados y en $74.7 \%$ el modo de transmisión. era sexual. En cuanto a la actividad física, el 57,0\% eran activos o irregularmente activos y el 67,5\% no tenían EM. Entre los activos, el 31,1\% tenía SM y entre los inactivos el 34,2\%, lo que no representó una asociación estadísticamente significativa (OR 0,87; IC 95\% 0,52 - 1,46; p = 0,595). Conclusión: Desde esta perspectiva, la actividad física no fue un factor protector para la EM en (PVVIH) que usaban terapias antirretrovirales (ART).

Palabras clave: SIDA; Dislipidemia; Terapia antirretroviral; Ejercicio Físico.

\section{Introdução}

O vírus do HIV (Human Imunodeficience Virus) é um retrovírus da subfamília dos Lentiviridae responsável por causar a AIDS, como todo vírus, este também tem tropismo por uma célula especifica do hospedeiro, no caso do HIV, as células de defesa do organismo (macrófagos e linfócitos TCD4). Os vírus são parasitas intracelulares obrigatórios, quando o HIV se liga a célula, que após o contato, desfaz a conformação de sua membrana permitindo que o capsídeo infiltre-se no citoplasma celular liberando, posteriormente, seu material genético, composto por RNA e a enzima transcriptase reversa, ao núcleo celular (Ministério da saúde, 2019). A transcriptase reversa faz com que o RNA viral (fita simples) converta-se em DNA viral (dupla fita) que posteriormente migra para dentro do núcleo celular onde sofre clivagens especiais para combine-se ao DNA do hospedeiro através da enzima integrase iniciando a replicação e produção de proteínas virais. Após todo o processo de reprodução, o vírus do HIV rompe o linfócito em busca de novas células para infecção (Rodrigues, 2017).

Em 2019, segundo a UNAIDS, existem em todo o mundo, cerca de 37,9 milhões de pessoas vivendo com HIV/AIDS (PVHIV), sendo que ocorreram 1,7milhões de novas infecções pelo vírus só neste ano (UNAIDS, 2019). No Brasil existiam, num total acumulado de 1980 a 2018, 926.742 PVHIV, sendo 606.936 (65,49\%) homens e 319.682 (34,51\%) mulheres. Do total de casos registrados neste período, 479.989 (51,79\%) são da região sudeste, seguido da região sul com 185.363 (20\%), $146.141(15,76 \%)$ na região nordeste, 56.119 (6,05\%) no centro-oeste e $59.129(6,38 \%)$ na região norte, sempre observando a predominância masculina dos indivíduos portadores. O perfil desta população varia conforme a região estudada, de modo geral, a nível nacional, estes indivíduos são homens, heterossexuais, com escolaridade igual ou inferior ao ensino fundamental completo (Brasil, 2018).

O número de infecções causadas pelo vírus do HIV no período 1980-2018 no estado do Paraná foram num total de 45.443 correspondendo a $24,51 \%$ dos casos registrados na região sul e 4,9\% dos casos registrado no Brasil. No município de Ponta Grossa, onde o estudo foi realizado, num total acumulativo de 1980 a 2018, o número de PVHIV era de 1681 (3,69\%), sendo $1004(59,72)$ indivíduos do sexo masculino e 677 (40,28\%) do sexo feminino, afirmando mais uma vez a predominância 
do comportamento de risco ligada ao sexo masculino, (Brasil, 2018).

As PVHIV têm seu sistema imunológico comprometido pelo dano causado às células de defesa, podendo causar a perda total da imunidade celular (Roit \& Delves, 2004). A fim de diminuir os danos causados pelo vírus do HIV, foram desenvolvidas drogas que atuam impedindo sua ação nas células de várias formas. Os inibidores nucleosídeos da transcriptase reversa (Lamivudina 3TC, Tenofovir TDF, Zidovudina AZT) atuam sobre a enzima transcriptase reversa tornando defeituosa a cadeia de DNA viral e impedindo a multiplicação do vírus, os Inibidores não nucleosídeos da transcriptase reversa (Efavirenz EFZ) agem diretamente na enzima transcriptase reversa bloqueando sua ação e a multiplicação do vírus, inibidores de protease (Atazanavir ATV) agem na enzima protease impedindo a replicação do vírus, Inibidores de fusão (Enfuvirtida T20) impede que o vírus se conecte a célula evitando assim sua multiplicação; Inibidores da Integrase (dolutegravir DTG) atua sobre a ensima intergrase, que age na integração do DNA viral com o DNA celular impedindo a replicação do vírus e evitando assim a infecção de novas células, inibidores de entrada (Maraviroc MRV) atua nos receptores localizados na membrana das células de defesa (macrófagos e linfócitos) impedindo a conexão do capsídeo viral com a célula e assim evitando a infecção das células de defesa (Parana, 2019).

As PVHIV devem ser tratadas com as terapias antirretrovirais (TARVs). Estas terapias, com o passar do tempo, podem ter complicações metabólicas como dislipidemia, lipodistrofia, hiperglicemia, hipertensão arterial (HAS), e a SM induzida pelo uso diário das TARVs (Oh et al., 2017).

A SM e definida como um conjunto de três de cinco fatores elencados para defini-la, os critérios para definição incluem obesidade central, circunferência abdominal (CA) maior que trina e cinco polegadas $(88,9 \mathrm{~cm})$ na mulher e quarenta polegadas $(101,6 \mathrm{~cm})$ no homem, HAS, pressão arterial sistólica maior que $130 \mathrm{mmhg}$ e/ou pressão arterial diastólica maior 85 mmhg ou fazer uso de medicamentos hipotensores para o controle PA, glicemia sérica de jejum aumentada (maior que 100 $\mathrm{mg} / \mathrm{dl}$ ) ou estar tomando hipoglicemiantes, triglicerídeos séricos maior $150 \mathrm{mg} / \mathrm{dl}$ ou estar tomando medicamentos para controle dos triglicerídeos , colesterol HDL (high density lipoprotein) sérico menor $40 \mathrm{mg} / \mathrm{dl} \mathrm{em}$ homens e menor que $50 \mathrm{mg} / \mathrm{dl}$ em mulheres ou estar tomando medicamentos para controle do colesterol (American Heart Association, 2015).

A prevalência da SM é um dado complexo de ser estimado devido a deficiência de dados no país (Neves; Mesquita, 2018). Alguns estudos apontam maior ocorrência em países desenvolvidos e em desenvolvimento e é observada com mais frequência em mulheres, indivíduos com baixa escolaridade, sedentárias ou que pouco praticam atividade física, diagnosticados com diabetes mellitus tipo dois (Leitão \& Martins, 2012). Devemos observar que a SM também tem sido encontrada em crianças e adolescentes devido ao estilo de vida e a alimentação baseada em carboidratos e ácidos graxos saturados, (Carvalho, Belém \& Oda, 2017).

Apesar da SM estar em foco no cenário atual da saúde, seus dados de prevalência e incidência são carentes, não há estudos de abrangência nacional com dados específicos de prevalência e incidência. Há alguns estudos pontuais, no Brasil, que corroboram com achados em outros apenas estudos (Neves; Mesquita, 2018; Haab; Benvegnð; Fische, 2019)

Além dos fatores fisiológicos, há também os fatores ambientais e sociodemográficos, que incluem alimentação e pratica de exercício físico, fatores hereditários e pessoas em uso de medicação de uso continuo, como as PVHIV em uso de TARVs (Moutinho; Pretto; Moreira, 2015)

Existem vários estudos mostrando que o exercício físico é um fator protetor para hipertensão, dislipidemias e diabetes tipo dois, que estão relacionados a síndrome metabólica, principalmente em indivíduos com alto risco para o desenvolvimento da SM (Lee; Ryu; Sung, 2018, Colombo et al., 2013). Coelho-Júnior et al. (2018) mostrou no seu estudo que uma rotina de exercícios físicos melhorou significativamente os níveis de pressão arterial, já Olea et al. (2017), foi mais além, demonstrou que, num período de 8 semanas, o programa de exercício aplicado, foi capaz reduzir em 100\% e normalizar 73\% os níveis pressóricos e melhorar os níveis glicêmicos dos indivíduos. 
Sabe-se que a prática de atividade física é um fator protetor para o desenvolvimento da SM em qualquer indivíduo, porem esta associação ainda não está clara no PVHIV e em uso de TARVs. Desta forma, o objetivo do presente estudo foi avaliar a associação entre o nível de atividade física e a SM em PVHIV em uso de TARV no município de Ponta Grossa.

\section{Metodologia}

Trata-se de um estudo transversal de abordagem quantitativa (Pereira et al, 2018) que faz parte de uma pesquisa longitudinal mais ampla em PVHIV no município de Ponta Grossa (Rodrigues, 2019). A abordagem foi quantitativa desenvolvida com uma amostra não probabilística de 265 PVHIV selecionados aleatoriamente no serviço de assistência especializada (SAE), no município de Ponta Grossa-Paraná no período de março de 2018 a maio de 2019.

As variáveis idade, sexo, história da infecção pelo HIV (data do diagnóstico, uso de antirretroviral as, classes de antirretroviral, data de exposição à terapia) e dados laboratoriais dos últimos três meses (HDL - c, GJ, TGL) foram obtidas do prontuário médico dos participantes fornecidos pelo SAE. Os dados clínicos pressão arterial (PA) e circunferência abdominal (CA) foram aferidos na triagem para consulta médica; foram utilizados como critérios de inclusão: PVHIV em uso de TARVs há no mínimo seis meses e com idade igual ou superior a dezoito (18) anos, ambos os sexos, alfabetizados, que concordassem em assinar um termo de consentimento livre e esclarecido (TCLE) e acompanhados pelo SAE do município. Os critérios de exclusão foram: indivíduos sem adesão ao tratamento antirretroviral e os participantes que não concordarem com a realização da pesquisa.

Para avaliar o nível de atividade física das PVHIV, foi aplicada uma versão curta do questionário de avaliação de atividade física (IPAQ). O IPAQ foi desenvolvido com a ideia de mensurar a quantidade de tempo de exercício físico realizado por um indivíduo. Foram avaliados os níveis de atividade física realizado por cada indivíduo, sendo classificados em sedentários, ativos e muito ativos conforme os critérios do IPAQ e classificados pelos autores em ativos e não ativos para uma segunda forma de avaliação. Além do tempo de exercício físico realizado por cada indivíduo também foram avaliados idade, sexo e comportamento sexual, etnia, escolaridade, estada civil, tempo de infecção e presença ou não de SM, segundo o critério do NCEP-III.

A análise estatística foi realizada com estatística descritiva com estimativa de frequência simples e relativa das variáveis qualitativas e média e desvio padrão das variáveis quantitativas. Para avaliar a associação entre o nível de atividade física e as variáveis de interesse foi utilizado o teste de qui-quadrado e a intensidade da associação com a OR (odds ratio) e intervalo de confiança de $95 \%$. Os testes foram considerados significativos quando $\mathrm{p}<0,05$.

Todos os voluntários foram devidamente informados sobre os procedimentos e objetivos deste estudo, após concordarem, assinaram um TCLE. A pesquisa não recebeu financiamento para a realização e não houve conflito de interesses. O estudo foi aprovado pelo comitê de ética em pesquisa da Universidade Estadual de Ponta Grossa (UEPG) parecer número 2.305.903.

\section{Resultados}

Na Tabela 1 estão descritas as variáveis sociodemográficas das PVHIV. A tabela demonstra de mais relevante que 44,5\% dos pacientes tinham ensino fundamental (completo ou incompleto), 41,1\% renda familiar de 1 a 2 salários mínimos, 40,4\% viviam em união estável ou casados, 56,2\% do sexo biológico masculino, 61,9\% declararam-se de etnia branca, 74,7\% o modo de transmissão foi sexual e 71,3\% eram heterossexuais. 
Tabela 1: Análise descritiva das variáveis escolaridade, renda categorizada, estado civil, sexo biológico, etnia, modo de transmissão e comportamento sexual em PVHIV em Ponta Grossa, Paraná, 2018.

\begin{tabular}{llrr}
\hline & & $\mathbf{N}$ & $\mathbf{\%}$ \\
\hline \multirow{4}{*}{ Escolaridade } & Analfabeto & 6 & $2,3 \%$ \\
& Ensino fundamental & 18 & $44,5 \%$ \\
& Ensino médio & 102 & $38,5 \%$ \\
& Ensino superior & 39 & $14,7 \%$ \\
\hline \multirow{3}{*}{ Renda } & Acima de 5 SM & 8 & $3,0 \%$ \\
& Até 1 SM & 88 & $33,2 \%$ \\
& De 1 a 2 SM & 109 & $41,1 \%$ \\
& De 3 a 5 SM & 60 & $22,6 \%$ \\
\hline \multirow{4}{*}{ Estado civil } & Casado(a) ou união estável & 107 & $40,4 \%$ \\
& Separado(a) ou divorciado(a) & 37 & $14,0 \%$ \\
& Solteiro(a) & 86 & $32,5 \%$ \\
\multirow{2}{*}{ Sexo biológico } & Viúvo(a) & 35 & $13,2 \%$ \\
\hline \multirow{3}{*}{ Etnia } & Feminino & 116 & $43,8 \%$ \\
& Masculino & 149 & $56,2 \%$ \\
\hline \multirow{2}{*}{ Modo de transmissão } & Outra & 164 & $61,9 \%$ \\
& Branca & 2 & $0,8 \%$ \\
& Indígena & 99 & $37,4 \%$ \\
\hline & Parda ou preta & 8 & $3,0 \%$ \\
Comportamento sexual & Heterossexual & 59 & $22,3 \%$ \\
& Homossexual & 198 & $74,7 \%$ \\
\hline & Ignorado & 7 & $2,6 \%$ \\
& & 189 & $71,3 \%$ \\
& Bissexual & 41 & $15,5 \%$ \\
& & 28 & $10,6 \%$ \\
\hline
\end{tabular}

Fonte: Autores.

A Figura 1 trata-se do gráfico de barras da frequência de atividade física dos pacientes. Verificou-se quanto a atividade física que 57,0\% dos pacientes eram ativos ou muito ativos, 28,7\% irregularmente ativos e 14,3\% sedentários, conforme os critérios predefinidos pelo IPAQ (Figura 1).

Figura 1: Gráfico de barras da frequência do nível de atividade física conforme critérios do IPAQ em pacientes vivendo com HIV/AIDS em Ponta Grossa, Paraná, 2018.

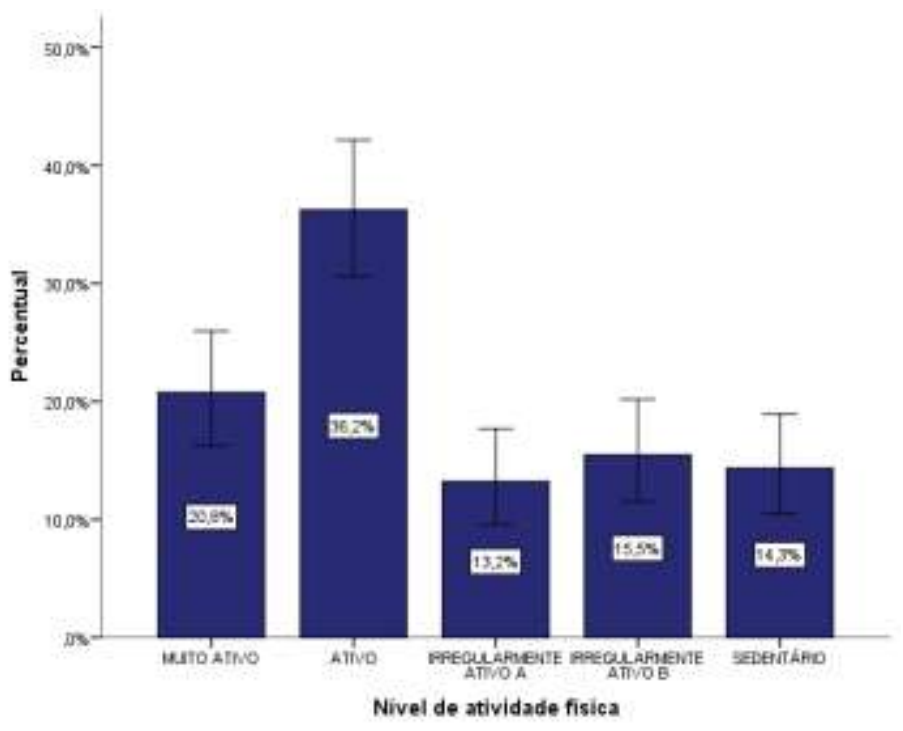

Fonte: Autores. 
A Tabela 2 descreve os dados referentes aos níveis de atividade física segundo os critérios do IPAQ e a associação com a presença de síndrome metabólica. Pôde-se observar que atividade física não pôde ser considerado um fator protetor para SM nas PVHIV avaliadas, não houve significância na comparação entre grupos. Entre os ativos e muito ativos, a prevalência de síndrome metabólica foi de $39,5 \%$ e $32,3 \%$, respectivamente e entre os sedentários foi de $29,1 \%$, o que não caracterizou diferença estatisticamente significativa.

Tabela 2: Frequências relativas do nível de atividade física conforme critérios do IPAQ em relação a síndrome metabólica em pacientes vivendo com HIV/AIDS em Ponta Grossa, Paraná, 2018.

\begin{tabular}{|c|c|c|c|c|c|c|}
\hline & \multicolumn{5}{|c|}{ Síndrome metabólica } & \multirow{3}{*}{ p-valor } \\
\hline & \multirow{2}{*}{$\begin{array}{l}\text { SIM } \\
\text { N \% }\end{array}$} & \multicolumn{2}{|c|}{ NÃO } & \multirow{2}{*}{$\begin{array}{c}\text { TOTAL } \\
\text { N \% } \\
\end{array}$} & \multirow[t]{2}{*}{ OR (IC95\%) } & \\
\hline & & $\mathbf{N}$ & $\%$ & & & \\
\hline ATIVO & 1539 , & 623 & 60 , & $5 \% 38100$, & REFERÊNCIA & \\
\hline Nível deIRREGULARMENTE ATIVO A & 720 , & 628 & 80 & $\% 35100$ & $0,63(0,26-1$, & 0,298 \\
\hline atividade IRREGULARMENTE ATIVO B & 1741, & 624 & 58 , & $5 \% 41100$, & $61,64(0,59-4$, & 0,338 \\
\hline física $\quad$ MUITO ATIVO & 3132 , & 7665 & 67 & $7 \% 96100,($ & $0,58(0,25-1, ?$ & 0,209 \\
\hline SEDENTÁRIO & 1629 , & 739 & 70 , & $9 \% 55100,($ & $0,86(0,42-1$, & 0,683 \\
\hline
\end{tabular}

Fonte: Autores.

\section{Discussão}

Para a criação dos grupos de nível de atividade física no presente estudo, foi utilizado como instrumento a versão curta do IPAQ. O IPAQ é um instrumento de avalição bem aceito em pesquisas relacionadas a prática do exercício físico para todas as faixas etárias e sem diferenciação de gênero. Garcia et al, afirma em seu trabalho que o IPAQ é um questionário válido para avaliação de exercício físico de modo habitual em adultos. Outro estudo realizado por Lima et al, (2019), demonstra que o IPAQ é o questionário mais utilizado para avaliação de adolescentes (28\%), porém necessita de uma padronização de um instrumento para melhor avaliação tendo em vista que alguns estudos utilizam instrumentos específicos para suas regiões de pesquisa. Já Rabacow et al. (2006) verificou que apenas dois dos questionários avaliados, foram validados, e ainda, que o IPAQ se apresenta como opção mais adequada aos idosos brasileiros. Tendo em vista que todos os estudos analisados apontam o IPAQ com um questionário para avaliação de atividade física válido, este foi selecionado para avaliação das PVHIV na cidade de Ponta Grossa.

Em relação ao perfil epidemiológico das PVHIV em Ponta Grossa, o presente estudo corrobora com os demais estudos já publicados referente ao assunto. O perfil de outros estudos no Brasil foi de pacientes masculinos heterossexuais, com média entre 20 e 40 anos e baixa escolaridade, semelhantes ao encontrado no presente estudo (Menezes et al., 2018; Moura; Faria, 2017; Silva et al., 2016.). No que diz respeito a coloração da pele/raça, já foi descrito na literatura que os dados podem não ser fidedignos, pois depende ou da autodeclaração ou de como profissional entrevistador o classifica, sendo assim uma variável com alta possibilidade de viés (Araújo; Bertolini; Bertolini, 2015).

No que diz respeito a declaração de orientação sexual, nenhum trabalho encontrado foi específico quanto à possibilidade de omissão da real orientação sexual dos indivíduos entrevistados, porém, no presente estudo, levantou-se a hipótese de possível viés desta declaração, haja vista que no contexto do HIV/AIDS há relatos de discriminação da pessoa nessa condição. Garbin et al. (2017) descreve em seu estudo que a discriminação da PVHIV ocorre de forma velada e em todas as classes sociais e a maioria dos envolvidos no estudo tinham a ideia que era mais fácil a contaminação pelo vírus HIV que pelo vírus da hepatite B e C. Os dados deste estudo mostraram que 80,7\% dos entrevistados, achavam mais fácil se contaminar com o vírus do HIV que o da hepatite B, e ainda 91,3\% dos entrevistados neste mesmo estudo, disseram que uma PVHIV deve 
fazer o tratamento em casa, $86 \%$ acreditavam ser possível contaminar-se com o vírus do HIV se o cirurgião dentista fosse uma PVHIV e a reciproca também e valida, pois há também uma recusa da parte dos profissionais da saúde (dentistas, citados no trabalho) em atender PVHIV, muitas vezes por ter pouco conhecimento quanto as formas de transmissão da doença.

É sabido na literatura que o exercício físico é benéfico a saúde e atua como fator protetor para SM em indivíduos saudáveis e que convivem com vírus do HIV/AIDS. Golvêa e Silva et al, (2015) descrevem em seu estudo um maior acumulo de gordura associada a baixas taxas metabólicas em PVHIV sedentários em relação ativos fisicamente, além disso, os praticantes de atividade física apresentaram menor índice de massa gorda, maior taxa metabólica e maior força. No que diz respeito a qualidade de vida, nota-se que os PVHIV e que são ativos fisicamente, apresentam maior qualidade de vida em relação aos sedentários, dados similares foram encontrados por Santos et al, (2014). No que tange a busca pela associação específica entre o nível de atividade física e a SM em PVHIV, não há outros estudos evidenciando este aspecto.

A associação indireta já foi pesquisada associando atividade física às dislipidemias, os resultados apresentados nos estudos utilizados, apresentam similaridade nos seus resultados, após um programa de atividade física, todos os indivíduos submetidos a ele, apresentaram melhora nos padrões lipídicos e na qualidade de vida (Soares et al., 2018; Eller et al., 2017).

Estudos apresentam resultados positivos para a associação de dislipidemias com o uso de TARVs. Um estudo realizado por Ceccato et al. (2011) demonstrou que o tratamento e o tempo de uso das medicações estavam diretamente associados as dislipidemias. Souza Neto de et al. (2013) evidencia em seu estudo que o perfil lipídico dos indivíduos em uso das TARVs pode ser melhorado com a pratica de atividade física e reeducação alimentar associada a terapia medicamentosa.

\section{Conclusão}

Apesar de haver evidências fisiológicas e na literatura de que o exercício físico pode proteger também o PVHIV da ocorrência da síndrome metabólica, o presente estudo não encontrou associação significativa entre os mesmos e a prevalência de síndrome metabólica entre ativos e muito ativos foi numericamente superior do que os irregularmente ativos ou sedentários.

Como trabalhos futuros, propõe-se um acompanhamento de PVHIV com diferentes níveis de atividade física e em tratamento para que possa contribuir melhor na elucidação da problemática envolvida com a prevalência e possibilidades de da síndrome metabólica.

\section{Referências}

American Heart Association (2015). What Is Metabolic Syndrome? https://www.heart.org/-/media/files/health-topics/answers-by-heart/what-is-metabolicsyndrome-300322.pdf?la=en\&hash=3B60478685B71C2CD6CEB93782DAE8B7EAD33445

Araújo, A. P. S.; Bertolini, S. M. M. G. \& Bertolini, D. A. (2015). Perfil epidemiológico e imunológico de idosos infectados pelo vírus da imunodeficiência humana. Estudos Interdisciplinares Sobre o Envelhecimento, 20 (1), 121-138.

Carvalho, A. R. M.; Belém, M. O. \& Oda, J. Y. (2017). Sobrepeso e obesidade em alunos de 6-10 anos de escola estadual de Umuarama/Pr. Arquivos de Ciências da Saúde da Unipar, 21 (1), 3-12. http://dx.doi.org/10.25110/arqsaude.v21i1.2017.6070.

Ceccato, M. G. B.; Bonolo, P. F.; Souza Neto, A. I.; Araújo, F. S. \& Freitas, M. I. F. (2011). Antiretroviral therapy-associated dyslipidemia in patients from a reference center in Brazil. Brazilian Journal of Medical and Biological Research, 44 (11), 1177-1183. http://dx.doi.org/10.1590/s0100-879x2011007500129

Coelho-Júnior, H. J. et al. (2018). Multicomponent exercise decreases blood pressure, heart rate and double product in normotensive and hypertensi ve older patients with high blood pressure. Archivos de Cardiología de México, 88 (5), 413-422. http://dx.doi.org/10.1016/j.acmx.2018.01.001

Colombo, C. M. et al. (2013). Efeitos de curto prazo de um programa de atividade física moderada em pacientes com síndrome metabólica. Einstein (são Paulo), 11 (3), 324-330. http://dx.doi.org/10.1590/s1679-45082013000300011.

Eller, O. C. W. S. et al. (2017). Controle das dislipidemias através do tratamento não farmacológico. Journal of Biology \& Pharmacy and Agricultural Management, 13 (3), 1-5.

Farias, C. R. L. et al. (2018). Persistent metabolic syndrome and risk of cardiovascular disease in children and adolescents. Revista Brasileira de Enfermagem, 71 (3), 1013-1021. http://dx.doi.org/10.1590/0034-7167-2016-0564. 
Garcia, L. et al. Validação de dois questionários para a avaliação da atividade física em adultos. Revista Brasileira de Atividade Física \& Saúde, 18 (3), $317-$ 318. http://dx.doi.org/10.12820/rbafs.v.18n3p317

Haab, R. S.; Benvegnð, L. A. \& Fische, E. V. Prevalência de Síndrome Metabólica em uma área rural de Santa Rosa. Revista Brasileira de Medicina de Família e Comunidade, 9 (90), 7-23.

Lee, J. Y.; Ryu, S. \& Sung, K. C. (2018). Association of baseline level of physical activity and its temporal changes with incident hypertension and diabetes mellitus. European Journal of Preventive Cardiology, 25 (10), 1065-1073.

Leitão, M. P. C. \& Martins, I. S. (2012). Prevalência e fatores associados à síndrome metabólica em usuários de Unidades Básicas de Saúde em São Paulo SP. Revista da Associação Médica Brasileira, 58 (1), 60-69. http://dx.doi.org/10.1590/s0104-42302012000100016

Menezes, A. M. F. et al. (2018). Perfil epidemiológico das pessoas soropositivas para hiv/aids. Revista de Enfermagem Ufpe on Line, 12 (5), 1225-1232. http://dx.doi.org/10.5205/1981-8963-v12i5a230907p1225-1232-2018

Ministério da Saúde-Brasil (2019). O que é HIV: doenças de condições crônicas e infecções sexualmente transmissíveis. http://www.aids.gov.br/pt-br

Moura, J. P. \& Faria, M. R. (2017). Caracterização e perfil epidemiológico das pessoas que vivem com HIV/AIDS. Revista de Enfermagem Ufpe on Line, 11 (12), 5214-5220 http://dx.doi.org/10.5205/1981-8963-v11i12a22815p5214-5220-2017

Moutinho, A. B. A.; Pretto, A. D. B. \& Moreira, A. N. (2015). Evolução do estudado nutricional de pacientes com AIDS atendidos em um ambulatório de nutrição. Sociedade Brasileira de Atividade Física e Saúde, 9 (51), 85-95.

Neves, M. M. \& Mesquita, M. M. (2018). Incidence of metabolic syndrome in patients using the services of the clinical laboratory of PUC of Goiás state. Revista Brasileira de Análises Clínicas, 50 (2), 139-143. http://dx.doi.org/10.21877/2448-3877.201800738

Oh, D. H. et al. (2017). Metabolic Complications among Korean Patients with HIV Infection: The Korea HIV/AIDS Cohort Study. Journal of Korean Medical Science, 32 (8), 1268-1273, http://dx.doi.org/10.3346/jkms.2017.32.8.1268

Olea, M. A. et al. (2017). Entrenamiento interválico de alta intensidad contribuye a la normalización de la hipertensión arterial. Revista Médica de Chile, 145 (9), 1154-1159. http://dx.doi.org/10.4067/s0034-98872017000901154

Paraná. Ministério da Saúde. Medicamentos Anti-HIV: quais são os antirretrovirais. http://giv.org.br/HIV-e-AIDS/Medicamentos/index.html

Rabacow, F. M. et al. (2006). Questionários de medidas de atividade física em idosos. Revista Brasileira de Cineantropometria \& Desempenho Humano, 4 (8), 99-106.

Moutinho, A. B. A.; Pretto, A. D. B. \& Moreira, A. N. (2015). Evolução do estado nutricional de pacientes com AIDS atendidos em um ambulatório de Nutrição. Revista Brasileira de Obesidade, Nutrição e Emagrecimento, 9 (51), 85-95. http://www.rbone.com.br/index.php/rbone/article/view/362

Pereira A.S. et al. (2018). Metodologia da pesquisa científica. UFSM. https://repositorio.ufsm.br/bitstream/handle/1/15824/Lic_Computacao_MetodologiaPesquisa-Cientifica.pdf?sequence $=1$

Rodrigues, N. F. (2017). Imunomodulação da replicação do HIV-1 pela hemaglutinina do vírus influenza. Tese de Doutorado, Instituo Oswaldo Cruz, Programa de Pós-Graduação em Biologia Celular e Molecular, Rio de Janeiro, Rio de Janeiro, Brasil.

Roitt, I. N. \& Delves, P. J. (2004). Fundamentos de Imunologia. Panamericana.

Soares, B. T. et al. Efeito do Exercício Físico Sobre a Dislipidemia. Revista de Atenção A Saúde, 16 (58), 12-16.

Souza Neto, A. I. et al. (2013). Dislipidemia e risco cardiovascular na terapia antirretroviral: o manejo dos fatores modificáveis. Revista Brasileira de Cardiologia, 26, 26-32. 